\title{
Enfermagem perioperatória e sua inserção nos planos de ensino das universidades
}

\author{
Perioperatory nursing and its insertion in university education plans \\ Enfermería perioperatoria y su inserción en los planes de enseñanza de las universidades \\ Mailma Costa de Almeida ${ }^{1 *}$, Nair Chase da Silva ${ }^{2}$, Aderlaine da Silva Sabino ${ }^{3}$.
}

\section{RESUMO}

Objetivo: Investigar a inserção da Enfermagem Perioperatória nos Planos de Ensino das Universidades de Manaus/AM. Método: Estudo descritivo-exploratório, qualitativo, baseado na análise categorial temática, em duas universidades públicas de Manaus/AM, com seis docentes que ministram conteúdos de Enfermagem Perioperatória. A produção foi obtida por meio de entrevista e grupo focal. Resultados: Os docentes eram do sexo feminino, com experiência na docência e assistência de Enfermagem Perioperatória; os planos de ensino permitem uma visão parcial da Enfermagem Perioperatória; de quatro disciplinas três possuem carga horária teórico-prática e apenas uma prática; nas ementas e conteúdos estão em duas disciplinas de maneira explicita e duas implícita; os objetivos constam em três disciplinas; o conteúdo estão direcionados a Enfermagem Perioperatória; as estratégias de ensino os docentes sentem a necessidade de trabalhar com metodologias inovadoras; os recursos de ensino são compatíveis com as estratégias de ensino; as referências bibliográficas situam-se entre 2005 a 2015 e período anterior a 2004, não se constatou a existência de periódicos, artigos científicos, teses e dissertações, sites e outros. Conclusão: O ensino da EP nas IES públicas esteve presente na formação do enfermeiro, entretanto, precisa de ações que qualifiquem na perspectiva de atender às DCN e saúde da população.

Palavras-chave: Ensino de enfermagem, Plano de ensino, Assistência de enfermagem perioperatória, Enfermagem em centro cirúrgico.

\begin{abstract}
Objective: To investigate the insertion of Perioperative Nursing in the Teaching Plans of the Universities of Manaus / AM. Method: Study descriptive-exploratory, qualitative, based on categorical thematic analysis, in two public universities of Manaus / AM, with six teachers who teach contents of Perioperative Nursing. The production was obtained through an interview and a focus group. Results: The teachers were female, with experience in teaching and assistance of Perioperative Nursing; the teaching plans allow a partial view of Perioperative Nursing; of four subjects have three theoretical-practical hours and only one practice; in the menus and contents are in two disciplines explicitly and implicitly two; the objectives are in three disciplines; the contents are directed to Perioperative Nursing; teaching strategies teachers feel the need to work with innovative methodologies; teaching resources are compatible with teaching strategies; the bibliographical references are between 2005 and 2015 and before 2004, the existence of periodicals, scientific articles, theses and dissertations, websites and others was not found. Conclusion: Conclusion: The teaching of PE in public $\mathrm{HEI}$ was present in the training of nurses, however, it needs actions that qualify with the perspective of attending the NCDs and health of the population.
\end{abstract}

Key words: Nursing teaching, Teaching plan, Perioperative nursing care, Nursing in a surgical center.

1Universidade do Estado do Amazonas (UEA), Manaus-AM. *E-mail: mcalmeida@uea.edu.br

${ }^{2}$ Universidade Federal do Amazonas (UFAM), Manaus-AM.

${ }^{3}$ Universidade Luterana do Brasil (ULBRA), Manaus-AM.

SUBMETIDO EM: 2/2019

ACEITO EM: 3/2019

PUBLICADO EM: 5/2019

REAS/EJCH | Vol. 11 (11) | e500 | DOI: https://doi.org/10.25248/reas.e500.2019 Página 1 de 9 


\section{RESUMEN}

Objetivo: Investigar la inserción de la Enfermería Perioperatoria en los Planes de Enseñanza de las Universidades de Manaus / AM. Método: Estudio descriptivo-exploratorio, cualitativo, basado en el análisis categorial temático, en dos universidades públicas de Manaus / AM, con seis docentes que ministran contenidos de Enfermería Perioperatoria. La producción fue obtenida por medio de una entrevista y un grupo focal. Resultados: Los docentes eran del sexo femenino, con experiencia en la docencia y asistencia de Enfermería Perioperatoria; los planes de enseñanza permiten una visión parcial de la Enfermería Perioperatoria; de cuatro disciplinas tres poseen carga horaria teórico-práctica y apenas una práctica; en los menús y contenidos están en dos disciplinas de manera explícita y dos implícita; los objetivos constan en tres disciplinas; el contenido está dirigido a la enfermería perioperatoria; las estrategias de enseñanza los docentes sienten la necesidad de trabajar con metodologías innovadoras; los recursos de enseñanza son compatibles con las estrategias de enseñanza; las referencias bibliográficas se sitúan entre 2005 a 2015 y período anterior a 2004, no se constató la existencia de periódicos, artículos científicos, tesis y disertaciones, sitios y otros. Conclusión: La enseñanza de la EP en las IES públicas estuvo presente en la formación del enfermero, sin embargo, necesita acciones que califiquen en la perspectiva de atender a las DCN y salud de la población.

Palabras clave: Enseñanza de enfermería, Plan de enseñanza, Asistencia de enfermería perioperatoria, Enfermería en centro quirúrgico.

\section{INTRODUÇÃO}

A enfermagem perioperatória e sua importância na formação profissional, está voltada a atender às demandas individuais, na fase em que o paciente se encontra no período anestésico-cirúrgico, isso faz com que haja necessidade de otimizar o processo de ensino que encontra-se voltado à globalização, através de uma formação de profissionais competentes e preparados (AVELAR MCQ; SILVA A, 2005).

Muitos fatores vêm preocupando os profissionais enfermeiros relacionados a formação para o cuidado perioperatório, que vão desde o ensino e aprendizagem até as ações que serão desenvolvidas (FONSECA RMP; PENICHE, ACG, 2009).

A assistência perioperatória abrange desde o pré-operatório, e é dividido em imediato que corresponde à indicação da cirurgia até o dia anterior desta e mediato que se refere às primeiras 24 horas que antecede ao procedimento cirúrgico. O transoperatório corresponde ao momento que o paciente entra no Centro Cirúrgico (CC) até o seu encaminhamento para a Sala de Recuperação Pós-anestésica (SRPA). O pós-operatório também é dividido em imediato que corresponde até as primeiras 24 horas depois do procedimento cirúrgico e mediato, período após as 24 horas e até sete dias depois relacionados a esta experiência cirúrgica (SOBECC, 2013).

Luvisotto MM et al. (2010) enfatiza que a equipe de enfermagem perioperatória deve promover a manutenção e recuperação da saúde do paciente cirúrgico, para isso é fundamental a supervisão e a avaliação sistemática do enfermeiro em cada etapa relacionada as ações da equipe, identificando os possíveis riscos decorrentes do processo anestésico cirúrgico, implantando e implementando normas e rotinas pautado nas boas práticas perioperatória.

A ausência da avaliação do enfermeiro perioperatório pode ocasionar eventos adversos decorrentes da falha do cuidado prestado ao logo da internação do paciente, que resultará em infecção de sítio cirúrgico, posicionamento inadequada, procedimento no local errado, administração incorreta de medicamentos e intercorrências no processo anestésico cirúrgico (BOHOMOL E, TARTALI JÁ, 2013).

As dificuldades enfrentadas nos cursos de enfermagem das Instituições de Ensino de Saúde (IES), não podem aceitar que o ensino em Centro Cirúrgico continue sendo visto como um conteúdo de menor importância. Observa-se que estes conteúdos estão inseridos nos currículos das instituições de várias formas, algumas oferecem de forma superficial, outras vezes, são substituídas por apenas visita na unidade do CC, 
o que dificulta o aluno a desenvolver suas habilidades. Ademais, tem-se a falta de docentes com experiência em CC para ministrar os conteúdos, sendo ainda um dos grandes problemas (TURRINI RNT, et al., 2012).

Nesse sentido, se faz indispensável desenvolverem competências e habilidades por meio de cursos de férias e voluntariados para suprir a necessidade de complemento de carga horária nos currículos de enfermagem das universidades (WORD RP, SAYLON C, 2002; SIGSBY LM, 2008).

Entende-se que a enfermagem bem fundamentada cientificamente muito pode fazer para minimizar os danos ao paciente nesse momento delicado de sua vida. Diante desse cenário elegeu-se como objeto de estudo a formação dos profissionais de enfermagem para atuação nessa área. Sendo assim, esta pesquisa tem o ensino da enfermagem perioperatória em IES públicas como foco de investigação.

Desse modo, diante das assertivas o estudo vislumbra-se ser capaz de propor alternativas para qualificação do ensino-aprendizagem do acadêmico de enfermagem, ao de investigar como se tem dado a Enfermagem Perioperatória e sua Inserção nos Planos de Ensino das Universidades de Manaus/AM.

Com base no exposto, o objetivo deste estudo constituiu em investigar o ensino da Enfermagem Perioperatória das universidades públicas de Manaus/AM.

\section{MÉTODOS}

Estudo de caráter descritivo-exploratório, qualitativo, com fontes documentais e orais. Conforme Minayo MCS (Org) (2014) os estudos qualitativos permitem ao pesquisador aumentar sua experiência em torno de determinado problema, absorver as questões do significado e da intenção dos atos inerentes à relação e estrutura social como um todo, advindas de construções humanas.

Inicialmente, pretendeu-se trabalhar com quatro cursos, doze docentes, sendo três para cada IES, entretanto duas IES estiveram ausentes do grupo focal, sendo excluídas do estudo. Diante deste acontecimento, participaram seis docentes dos cursos de graduação em enfermagem, considerando como critério de inclusão: docentes que ministravam aula de Enfermagem Perioperatória nas disciplinas que abordam a temática no período da coleta de dados, ambos os sexos; está em condição de contribuir como estudo e de Exclusão: ausência durante a técnica do grupo focal. Os dados foram coletados por meio de análise de documentos, entrevista (MINAYO MCS (ORG), 2014) e grupo focal, realizado durante os meses de fevereiro a março de 2015 , conforme agendamento prévio com os docentes.

A análise documental deu-se nos planos de ensino das disciplinas que contemplavam os conteúdos de enfermagem perioperatória, compreendendo: os elementos que compuseram esses planos, a saber: identificação, ementas, objetivos, conteúdo, metodologias de ensino, avaliação e bibliografia.

Utilizou-se três instrumentos para o levantamento de dados: um questionário, um roteiro para análise de documento e um roteiro de debate para conduzir o grupo focal. Para delinear o perfil dos docentes, foi utilizado um questionário que continha informações para delinear o perfil dos que ministravam conteúdos de enfermagem perioperatória.

Para analisar os planos de ensino das IES estudadas, foi aplicado um roteiro que compreendeu: 1 . Identificação, 2. Ementas, 3. Objetivos, 4. Conteúdo Programático, 5. Estratégias de Ensino, 6. Recursos de ensino 7. Avaliação e Referência bibliográfica. Para descrever a opinião dos docentes foi utilizado um roteiro de debate para o grupo focal. A técnica de análise temática foi realizada em três etapas: pré-análise, exploração do material e tratamento dos resultados e interpretação.

Considerando a Resolução CNS no 466/12, o projeto foi encaminhado ao Comitê de Ética em Pesquisa (CEP) da Universidade Federal do Amazonas (UFAM). O estudo foi desenvolvido após a aprovação do CEP com CAEE 39414514.2.0000.5020 e autorização por meio da assinatura do Termo de Consentimento Livre e esclarecido (TCLE).

Os docentes foram identificados pela letra $D$ de docente e número seguido por uma ordem crescente. Assim, foram identificados como D1, D2... D6. 


\section{RESULTADOS}

Os resultados foram apresentados de modo sistematizados: perfil dos docentes da disciplina de enfermagem perioperatória; inserção da enfermagem perioperatória nos planos de ensino das IES estudadas; opinião dos docentes que ministravam conteúdos de enfermagem perioperatória. O perfil dos docentes revela predominância do sexo feminino sobre o sexo masculino, apresentando entre 06 a 28 anos de exercício no ensino de enfermagem e 01 a 28 anos de exercício na docência em enfermagem perioperatória (Tabela1).

Tabela 1 - Caracterização do perfil dos docentes quanto ao sexo, tempo de docência, tempo na assistência e na enfermagem perioperatória e tempo na docência e assistência em Enfermagem Perioperatória (EP).

\begin{tabular}{llllll}
\hline Docente & Sexo & Tempo de docência & Tempo de docência na EP & Tempo na assistência EP & Tempo \\
\hline 1 & $\mathrm{~F}$ & 12 anos & 12 anos & 19 anos & 19 anos \\
2 & $\mathrm{M}$ & 06 anos & 03 anos & 07 anos & 3 anos \\
3 & $\mathrm{~F}$ & 10 anos & 10 anos & 16 anos & 16 anos \\
4 & $\mathrm{~F}$ & 07 anos & 01 ano & 05 anos & 5 anos \\
5 & $\mathrm{~F}$ & 28 anos & 28 anos & 28 anos & 28 anos \\
6 & $\mathrm{~F}$ & 28 anos & 28 anos & 18 anos & 18 anos \\
\hline
\end{tabular}

Fonte: Dados da pesquisa, 2015.

Ainda na construção do perfil buscou-se saber se os docentes tinham exercício exclusivo no ensino e qualificação para atuar na graduação. Os resultados em relação a atuação dos docentes evidenciam que quatro atuam tanto na docência quanto na assistência; seis são pós-graduados (três especialistas, dois mestres e um doutor) (Tabela 2).

Tabela 2 - Caracterização dos docentes segundo atuação, titulação e área de qualificação.

\begin{tabular}{llll}
\hline Docente & Atuação & Titulação & Área \\
\hline 1 & Docente e Assistencial & Especialista & Urgência e Emergência \\
2 & Docente & Mestre & Mestrado em enfermagem \\
3 & Docente e Assistencial & Especialista & Urgência e Emergência \\
4 & Docente & Especialista & Enf. Médico-cirúrgica e urgência e Emergência \\
5 & Docente e Assistencial & Doutor & Ciências biológicas \\
6 & Docente e Assistencial & Mestre & Ciências do alimento \\
\hline
\end{tabular}

Fonte: Dados da pesquisa, 2015.

\section{A enfermagem perioperatória nos Planos de Ensino}

A enfermagem perioperatória está contida nas disciplinas: 1- Enfermagem na Atenção Integral a Saúde do Adulto, 2- Enfermagem no Processo de Saúde do Neonato, Criança e Adolescente, 3- Estágio Curricular Urbano e 4- Enfermagem na Atenção Integral a Saúde do Adulto e do Idoso II (Tabela 3).

Tabela 3 - Nomenclatura e distribuição de carga horária das disciplinas que apresentam conteúdos de enfermagem perioperatória.

\begin{tabular}{|c|c|c|c|c|c|c|c|}
\hline & \multirow[t]{2}{*}{ Disciplinas } & \multicolumn{3}{|c|}{$\begin{array}{l}\text { Carga Horária da } \\
\text { Disciplina }\end{array}$} & \multicolumn{3}{|c|}{$\begin{array}{c}\text { Carga Horária de Enfermagem } \\
\text { Perioperatória } \\
\end{array}$} \\
\hline & & Teórica & Prática & Total & Teórica & Prática & Total \\
\hline 1 & $\begin{array}{l}\text { Enfermagem na atenção integral à Saúde } \\
\text { do Adulto }\end{array}$ & 90 & 150 & 240 & 11 & 55 & 66 \\
\hline 2 & Estágio Curricular Urbano & -- & 390 & 390 & --- & 78 & 78 \\
\hline 3 & $\begin{array}{l}\text { Enfermagem no processo de saúde do } \\
\text { Neonato, criança e adolescente }\end{array}$ & 90 & 60 & 150 & 08 & --- & 08 \\
\hline 4 & $\begin{array}{l}\text { Enfermagem na atenção integral à saúde } \\
\text { do adulto e do idoso II }\end{array}$ & 90 & 60 & 150 & 84 & 60 & 144 \\
\hline
\end{tabular}

Fonte: Dados da pesquisa, 2015. 


\section{Carga Horária}

Das quatro disciplinas ministradas, três possuíam carga horária teórico-prática e uma, apenas carga horária prática, por se tratar do estágio curricular. Quando observado, a carga horária específica para enfermagem perioperatória era em torno de 10\% para as disciplinas Enfermagem na Atenção Integral à Saúde do Neonato, da Criança e Adolescente e na disciplina Enfermagem Integral à Saúde do Adulto e Idoso, cujas horas destinadas à enfermagem perioperatória correspondiam a 93\%.

\section{Ementas}

Das quatro disciplinas que abordavam conteúdos de enfermagem perioperatória, apenas duas apresentavam de forma explícita.

\section{Objetivos}

As disciplinas 1 e 4 contemplavam a enfermagem perioperatória em seu objetivo geral e, explicitamente, em quatro de seus objetivos específicos, podendo considerar-se ainda que tais conteúdos poderiam ser trabalhados nos demais objetivos, quando trata dos sistemas de classificação de enfermagem NANDA (Classificação dos diagnósticos de enfermagem), NIC (Classificação das intervenções) e NOC (Classificação dos resultados).

Nas disciplinas 2 e 3, a enfermagem perioperatória esteve presente de forma implícita e explícita. $\mathrm{Na}$ disciplina 2, a enfermagem perioperatória esteve implícita no objetivo geral, quando se referiu-se às funções assistenciais, gerenciais, educativas, integrativas e de pesquisa do enfermeiro e explícitas, quando se remeteu ao uso da SAE nas diferentes situações cirúrgicas, no pré, trans e pós-operatório. Na disciplina 3, os conteúdos da enfermagem perioperatória não foram referidos, podendo estar contidos nas expressões "assistência integral" e "nos diferentes níveis de atenção". A disciplina 4 apresentou maior quantidade de carga horária destinada à enfermagem perioperatória em relação às demais disciplinas.

\section{Conteúdo Programático}

O processo que direcionou esta análise dos dados ocorreu da leitura dos planos de ensino, identificando conteúdos importantes para formação do enfermeiro sobre a enfermagem perioperatória.

Quadro 1 - Conteúdos apresentados nas disciplinas em relação aos aspectos organizacionais da assistência de enfermagem perioperatória.

\begin{tabular}{|c|c|}
\hline Conteúdos & Disciplinas \\
\hline 1. Base histórica da enfermagem perioperatória no processo do cuidar do adulto. & 1,3 e 4 \\
\hline $\begin{array}{l}\text { 2. Planejamento, implementação e avaliação do cuidado no pré-operatório imediato, } \\
\text { pós-operatório, incluindo a verificação de exames laboratoriais e todos os cuidados } \\
\text { e preparo do paciente para o procedimento cirúrgico e cuidados no pós-operatório } \\
\text { imediato e mediato e complicações. }\end{array}$ & 1,3 e 4 \\
\hline $\begin{array}{l}\text { 3. Assistência de enfermagem em cirurgias do trato digestório, urológicas, } \\
\text { ginecológicas, oncológicas, ortopédicas, plásticas, neurológica, gerais e outras. }\end{array}$ & 2,3 e 4 \\
\hline
\end{tabular}

Fonte: Dados da pesquisa, 2015.

Ao analisar essa temática, observou-se que os aspectos organizacionais da enfermagem perioperatória estavam contidos majoritariamente nas disciplinas 1, 3 e 4 . Notou-se pouca participação da disciplina 2 nesse aspecto, quando o esperado era de que apenas no item 1 ela precisasse figurar. Os temas relacionados à atuação do enfermeiro no ambiente do centro cirúrgico estão apresentados no Quadro 2. 
Quadro 2- Disciplinas que abordavam os conteúdos relacionados à atuação do enfermeiro no ambiente do centro cirúrgico.

\begin{tabular}{|c|c|}
\hline Conteúdos & Disciplinas \\
\hline $\begin{array}{l}\text { 1. Assistência de enfermagem no pré-trans-pós no centro cirúrgico (enfermagem } \\
\text { perioperatória) }\end{array}$ & $1,2,3$ e 4 \\
\hline $\begin{array}{l}\text { 2. Cuidados no preparo com a pele do paciente na antissepsia e degermação antes } \\
\text { do procedimento cirúrgico }\end{array}$ & 1 e 4 \\
\hline 3. Indução anestésica e riscos & 1 e 4 \\
\hline 4. Monitorização & 4 \\
\hline $\begin{array}{l}\text { 5. Posicionamento do paciente adequado para o procedimento cirúrgico conforme a } \\
\text { cirurgia }\end{array}$ & 1 e 4 \\
\hline 6. Intercorrências durante o transoperatório & - \\
\hline 7. Transporte para recuperação anestésica & - \\
\hline 8. Recuperação anestésica & 1 e 4 \\
\hline 9. Cuidados na SRPA e complicações pós-operatória e pós-anestésica & $1 \mathrm{e} 4$ \\
\hline 10. Alta da sala de recuperação anestésica e transporte para clínica cirúrgica & 4 \\
\hline 11. Profilaxia das infecções de sítio cirúrgico e inserção de cateteres venosos & 4 \\
\hline 12. Cirurgia Segura & 1 \\
\hline 13. Riscos para equipe cirúrgica em relação aos materiais biológicos & - \\
\hline
\end{tabular}

Fonte: Dados da pesquisa, 2015.

Os itens 6, 7 e 13 não foram abordados por nenhum plano de ensino de maneira explícita, entretanto podem ter sido no item 1 .

\section{Estratégias de ensino}

Os métodos mais utilizados foram aulas expositivas e dialogadas, estudos dirigidos e trabalhos em grupos.

\section{Recursos de ensino}

Os planos de ensino das IES públicas de Manaus, apresentaram recursos compatíveis relacionados com as estratégias de ensino.

\section{Avaliação}

Do ponto de vista qualitativo, observou-se que a avaliação da enfermagem perioperatória ocorria por meio de testes, provas, estudo de caso, prática e seminários. Na perspectiva quantitativa, eram realizadas de 3 a 7 avaliações, entretanto, não é possível afirmar quantas dessas avaliações estavam relacionadas aos conteúdos de EP, uma vez que esses números se referiam às disciplinas nas quais a EP estava inserida. Observou-se que a avaliação alcançou aos objetivos, uma vez que em relação ao conhecimento, foi realizada prova discursiva e provas objetivas, em relação às habilidades: prova prática, para atitudes: prova discursiva. 


\section{Referências bibliográficas}

A análise destes documentos permitiu observar quantas referência bibliográficas básicas e complementares estavam direcionados à EP nos planos de ensino e ao ano das referências utilizadas.

As referências foram apresentadas no final do plano de ensino das IES, no entanto, se as mesmas estivessem distribuídas por unidades de ensino, ficariam mais preciso identificá-las de acordo com os conteúdos trabalhados. Após a análise, foi possível observar que os planos contêm referências básicas e complementares. As bibliografias complementares apresentaram-se diferentes nos planos, ou seja, nenhum plano utilizou referências iguais neste quesito, enquanto que as bibliografias básicas apenas uma se repetia em três planos de ensino.

A disciplina Enfermagem no Processo do Cuidar da Saúde do Adulto e do Idoso II apresentou mais referências direcionadas à $\mathrm{EP}$, utilizando como referência complementar o livro de práticas recomendadas pela Sociedade Brasileira de Enfermagem de Centro Cirúrgico, Recuperação Anestésica e Centro de Material e Esterilização (SOBECC). Neste apresenta conteúdos importantes sobre a EP e deteve mais horas práticas e teóricas direcionadas a estes conteúdos.

\section{DISCUSSÃO}

O perfil dos docentes indicou predominância do sexo feminino sobre o sexo masculino, embora o ingresso do masculino tenha crescido na área da enfermagem, corroborando com os estudos de Terra FS, et al. (2011) e Friedianter MR e Moreira MTA, (2008).

Em relação aos planos de ensino observou-se que nas IES os conteúdos referentes EP estavam presentes em um número pequeno de disciplinas, mas em nenhuma IES foi encontrada uma disciplina específica. Turrini RNT, et al. (2012), destacam que as escolas de enfermagem apresentam esses conteúdos de formas variadas, algumas com disciplinas específicas, outras encontravam-se em apenas uma pequena quantidade de conteúdo inserido em uma determinada disciplina e outras não ofereciam nenhum conteúdo durante o curso de graduação.

Um estudo realizado em 10 cursos de graduação de enfermagem, na cidade e região metropolitana de São Paulo, em 2002, avaliando a assistência de EP nos cursos de graduação, constatou que 70 tinham a disciplina de enfermagem em CC e Central de Material e 30\% estavam inseridos na disciplina Saúde do Adulto, o que contradiz com os resultados desta pesquisa (AVELAR MCQ, SILVA A, 2005).

Para Turrini RNT et al. (2012), a Universidade Australiana de Notre Dame é uma das poucas Universidades que tem uma disciplina de enfermagem perioperatória, embora apresentem carga horária prática-teórico pequena, mas considerada o suficiente para que o enfermeiro possa iniciar suas atividades no CC. Nesse contexto, a visão de Scarton G (2002), enfatiza que ementa é uma descrição discursiva que resume o conteúdo conceitual ou conceitual / procedimental de uma disciplina. Libâneo JC (1994) pondera que o professor promove esclarecimento e orientações pautada nos objetivos educacionais.

Assim, Leite AS e Turrini RNT (2014) identificaram que os assuntos mais abordados na referida disciplina foram: planejamento físico e recursos em CC; modelo de Sistematização de Enfermagem Perioperatória (SAEP), assistência de enfermagem no pré, trans e pós-operatório, riscos físicos e biológicos da equipe cirúrgica, antissepsia e degermação, posição cirúrgica, instrumentação cirúrgica, hemostasia (sutura e bisturi elétrico), anestesia, monitorização do paciente, recuperação anestésica, riscos e controle de infecção em CC, assistência ao paciente em cirurgia ambulatorial, avanços tecnológicos no CC (videocirurgias, robótica, laser e outros). De igual modo, o presente estudo identificou que nas IES públicas em Manaus os conteúdos sistematizaram em duas dimensões: a) aspectos organizacionais da assistência de EP; b) atuação do enfermeiro no ambiente do centro cirúrgico.

Os conteúdos relacionados à EP nos planos de ensino se fazem imprescindíveis para formação do enfermeiro havendo necessidade de oportunizar durante a vida acadêmica situações reais em todas as áreas (Leite AS e Turrini RNT (2014); SOUZA CS, et al., 2006).

REAS/EJCH | Vol. 11 (11) | e500 | DOI: https://doi.org/10.25248/reas.e500.2019 Página 7 de $\mathbf{9}$ 
Assim, Rodrigues J, et al. (2012), em estudo sobre o ensino do cuidado de enfermagem na disciplina Saúde Mental, no qual foram analisados os Projeto Pedagógico do Curso (PPC) e os planos de ensino de enfermagem psiquiátrica, evidenciaram que o uso de metodologias interativas aumenta a participação do aluno no processo de ensino e aprendizado.

Em outros estudos, as metodologias tradicionais ainda existem, mas estão caminhando para o uso de metodologias mais interativas, através das dinâmicas em grupos, estudos dirigidos e aulas dialogadas (MAYER DE e KRUSE MHL, 2003).

O uso de estratégias de ensino mais inovadoras ou interativas refere-se à ruptura de paradigmas dominantes, no qual existe migração do "ensinar" para o "aprender", valorizando o aprender a aprender e fazendo com que o aluno desenvolva autonomia individual relacionada às habilidades e à comunicação. No entanto, para que o aluno seja capaz de aprender a aprender, é necessário que as IES priorizem o uso de metodologias mais ativas, inovadoras e problematizadoras (SOUZA CS, et al., 2014).

Outro ponto de interesse, evidenciado na pesquisa é a necessidade de os recursos de ensino da EP se torne um instrumento facilitador para o aluno compreender o universo do centro cirúrgico através de materiais e equipamentos específicos para demonstração do ambiente perioperatório (FRIEDLANDER MR e MOREIRA MTA, 2006).

Os achados sobre a avaliação devem ser contínua, criando um "feedback" e possibilita ao professor avaliar o que o aluno falta aprender para alcançar os objetivos, as estratégias de avaliação que são descritas, como provas escritas discursivas e objetivas, práticas, orais, observação, entrevistas, questionários e diário de curso (GIL AC, 2013). Bastos ABB (2006) ainda coloca a importância da utilização de outras fontes de recursos para o aprendizado, como leitura de outros textos, como artigos de revistas científicas e jornais.

Dessa forma, a inserção da Enfermagem Perioperatória nos Planos de Ensino das Universidades de Manaus/AM pressupõe reflexões para novas articulações de saberes, não apenas voltado para valorização de conteúdos do componente curricular da matriz do curso, mas também para compreender a transdisciplinaridade de maneira mais sólida.

\section{CONCLUSÃO}

Os achados do estudo sobre a inserção da EP nos planos de ensino das universidades permitiu reflexões de modo a repensar o processo formativo do enfermeiro no que diz respeito as questões relacionadas a carga horária teórico prática, as ementas e conteúdo e metodologias inovadoras. A opinião dos docentes sobre o ensino da EP potencializou a análise, na medida em que dificuldades, facilidades, expectativas e sugestões foram expressas. Portanto, o ensino da EP nas IES públicas estão presentes na formação do enfermeiro, entretanto precisam de ações que qualifiquem na perspectiva de atender às $\mathrm{DCN}$ e as necessidades de saúde da população. Diante disso, ressalta-se a necessidade de desenvolver pesquisas científicas que estimulem uma discussão mais ampla de modo a contribuir significativamente no ensino da enfermagem perioperatória proporcionando um encandeamento solido e sistematizado para direcionar os conteúdos nos demais componentes curriculares, construindo novos caminhos e soluções para possibilitar o desenvolvimento contínuo do processo de formação.

\section{REFERÊNCIAS}

1. AVELAR, MCQ, SILVA, A. Assistência de enfermagem perioperatória: ensino em cursos de enfermagem. Rev Esc. Enferm. USP. V. 39, n.1, p. 46-52. 2005.

2. BASTOS ABB. Metodologias ativa. 2006. Disponível em: http://educacaoeme dicina.blogspot.pt/2006/02/metodologias-ativas.html. Acesso em: 04 de outubro 2015.

3. BOHOMOL E, TARTALI JA. Eventos adversos em pacientes cirúrgicos: conhecimento dos profissionais de enfermagem. Acta Paul Enferm. 2013;26(4)

4. FONSECA R MP, PENICHE ACG. Enfermagem em centro cirúrgico: trinta anos após criação do Sistema de Assistência de Enfermagem Perioperatória. Acta Paul. Enf. v. 22, n. 4, p. 428-461, 2009. 
5. FRIEDLANDER MR, MOREIRA MTA. Formação do enfermeiro: características do professor e o sucesso. Rev. Bras Enfermagem, v. 59, n.1, p. 9-13, jan./fev. 2006.

6. GIL AC. Metodologia do ensino superior. 4 ed. São Paulo: Atlas, 2013.

7. LEITE AS, TURRINI RNT. Análise do ensino de enfermagem em centro cirúrgico nas escolas de são Paulo. Rev. Bras. Enferm. v. 67, n. 4, p. 512-519, jul./ago. 2014.

8. LIBÂNEO JC. Didática. São Paulo: Cortez. 1994.

9. LUVISOTTO MM, et al. Atividades assistenciais e administrativas do enfermeiro na clínica médicocirúrgica. Einstein. 2010;8(2):209-14.

10. MAYER DE, KRUSE MHL. Acerca de diretrizes curriculares e projetos pedagógicos: um início de reflexão. Rev. Bras. Enferm, Brasília (DF), v. 56, n.4, p.335-339, jul./ago. 2003.

11. MINAYO MCS (Org.). O desafio do conhecimento: pesquisa qualitativa em saúde. 14 ed. São Paulo: Hucitec, 2014.

12. RODRIGUES J, et al. Ensino do cuidado em saúde mental na graduação em enfermagem. Acta Paul Enferm. v.25, n.6, p. 844-51. 2012.

13. SCARTON G. Guia de produção textual: assim é que se escreve. Porto Alegre: PUCRS, FALE/GWEB/PROGRAD, [2002]. Disponível em: < http://www.pucrs.br/gpt >. Acesso em: 15 de setembro, 2015.

14. SIGSBY LM. A voluntary summer program to expose nursing students to the perioperative especialty. AOR J. v. 88 , n. 4 , oct. 2008.

15. SOCIEDADE BRASILEIRA DE ENFERMEIROS EM CENTRO CIRÚRGICO, RECUPERAÇÃO ANESTÉSICA E CENTRO DE MATERIAL E ESTERILIZAÇÃO. Práticas recomendadas. São Paulo: SOBECC, 2013.

16. SOUZA CS, et al. Estratégias inovadoras para métodos de ensino tradicionais - aspectos gerais. Medicina (ribeirão Preto), v. 47, n.3, p. 284-292. 2014.

17. TERRA FS, et al. Perfil dos docentes de cursos de enfermagem em de Universidades públicas e privadas. Rev. enferm. UERJ, Rio de Janeiro, v. 19, n.1, p. 26-33, jan./mar. 2011.

18. TURRINI RN. Ensino da enfermagem em centro cirúrgico nos cursos de bacharelado do Brasil. 2012. 216p. Tese (Doutorado). Escola de Enfermagem - Universidade de São Paulo. São Paulo, 2012.

19. TURRINI RNT, et al. Ensino de enfermagem em Centro Cirúrgico: transformações da disciplina na escola de enfermagem da USP (Brasil). Rev. Esc. enf. USP, v. 46, n.5, p.1268-1273, 2012.

20. WORD RP, SAYLOR C. Nursing School Curricula and Hospital-based Training Programs. AORN J. v.76, n. 6, p.1022-1031, dec. 2002. 